\title{
An Energy-efficient Grid based Clustering Topology for a Wireless Sensor Network
}

\author{
Ketki Ram Bhakare \\ Computer Science \& Engg \\ RCERT, Chandrapur \\ India.
}

\author{
R. K. Krishna \\ Electronics Engineering \\ RCERT, Chandrapur. \\ India.
}

\author{
Samiksha Bhakare \\ MCA Department \\ BCCE, Nagpur \\ India.
}

\begin{abstract}
Wireless Sensor Network (WSN) is an emerging technology. It is predicted that in future, WSN will change the human life totally. Energy optimization in Wireless Sensor Network (WSN)is one of the challenging issue. Wireless Sensor Network composed of a set of tiny sensor nodes. The nodes are continuously sense and transmit the data. WSN have a wireless nature, due to this has a limited lifetime. So increase the lifetime of Wireless Sensor Network and Minimize energy cost in wireless sensor network are an important problem. To solve this problem clustering technique are always used, among the entire clustering technique grid based clustering is more efficient. Grid based clustering is more simple and feasible, and has so much advantage with respect to other clustering method. In this we proposed a grid based clustering algorithm for minimizing energy consumption, where clustering is formed depending upon the interest of nodes.
\end{abstract}

\section{General Terms}

Wireless Sensor Network, Clustering,Grid Based Clustering

\section{Keywords}

Path Loss, Distance Distribution.

\section{INTRODUCTION}

Wireless Sensor Networks (WSNs) consist of small sensing nodes with capabilities- computation and wireless communications. There are several characteristics of wireless sensor nodes that limit their functionality. due to the use of batteries Wireless sensors often have limited energy. The total network capacity is limited by the use of unsophisticated wireless links that limit the range, reliability, and throughput of communication between nodes.

Wireless sensor network is consist of a set of tiny sensor nodes and sink nodes which has significant application in weather forecasting, medical monitoring, military target tracking and environmental detection. Sensor nodes transmit the data through multiple hops to the sink which is located far from the target. However, sensor nodes have a limited battery lifetime, so how to effectively save the energy of battery and increase the network lifetime has been the important research and issue in wireless sensor network. Sensor nodes have many modules; the communication module consumes the most electricity. The cost of communication is closely related to the way of routing protocol is set up. If routing protocol operates efficiently, the energy that every node consumes would be minimized. Therefore, it's desirable that the routing protocols should resolve issues like energy efficiency, load balance, minimum delay, etc. Especially, energy efficiency is the important key issue for keeping a longer network lifetime.
One of the advantages of wireless sensors networks (WSNs) is their ability to operate in harsh environments in which human monitoring schemes are difficult, inefficient and sometimes infeasible. Therefore, in such type of area sensors are deployed randomly by a relatively uncontrolled means, for e.g. dropped by a helicopter, and form a ad-hoc network collectively. Sensors node have a battery-powered, so that sensors have a limited lifetime and it get easily damaged during deployment in vast area. So to cover a given vast area a large number of sensors are expected in most WSNs applications. It is envisioned that hundreds or even thousands of sensor nodes will be involved. Designing and operating such large size network would require scalable architectural and good management strategies.

In addition, sensors in such environments are energy constrained and their batteries cannot be recharged. Therefore, for extending the lifetime of sensors, an important factor is to design a energy-aware algorithms.[5]

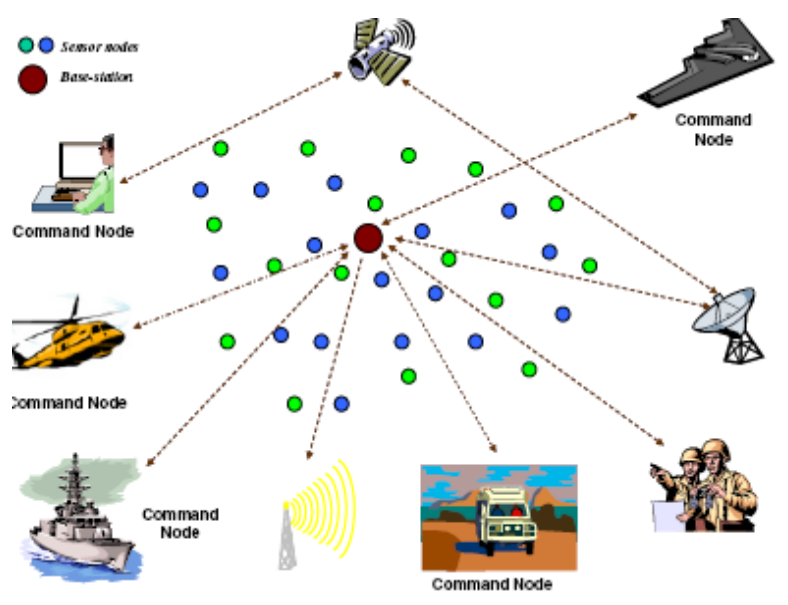

Fig 1: Architecture of WSN for a military application

In recent years, the major advances takes place in the development process of wireless sensors and IC process technology. Because of these advances, wireless sensor networks (WSNs) have been replacing traditional network technologies. These WSNs have a number of advantages over wired networks, such as ease of deployment, extended transmission range, and self-organization. There are, however, a few inherent limitations to WSNs. These include low communication bandwidth, small storage capacity, limited computation resources, and limited device energy. In terms of energy, many researchers assume that all nodes in a sensor network are battery-driven. 
Currently, WSNs are used in various applications. Figure 2 shows a schematic of applications for WSNs. Among their many applications, they can be used in the military, in agriculture, in transportation, in manufacturing, and in smart homes. [4]

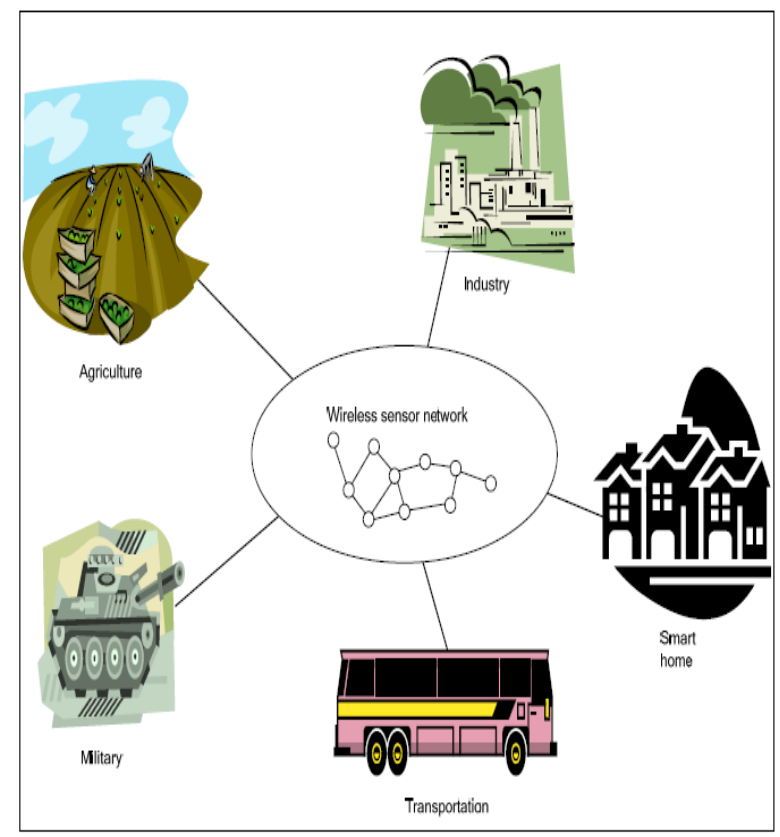

Fig 2: Wireless sensor network applications.

In wireless sensor network two most important issues are minimize the energy cost in wireless sensor network and Prolong lifetime of wireless sensor network. To solve this problem clustering technique are always used, among all the clustering technique grid based clustering is more efficient. Grid based clustering attracted lot of attention because of their scalability and simplicity. Even though the number of nodes increases grid based clustering gives the same performance when there is a limited number of nodes ,so it is scalable.

So in order to minimize energy consumption and prolong network lifetime in grid-based clustering schemes, an important problem is to determine the optimal grid size, Various gridding schemes have been proposed, but so far most existing work has used the average distance within a grid or between neighbor grids to calculate the energy consumption. However, we found that the average distance approach is not exact(accurate) and can largely underestimate the actual value due to the superliner path loss exponent of over the wireless transmissions.

\section{BACKGROUND \& RELATED WORK}

In wireless sensor network an efficient arrangement of network topology is required for energy optimization. Clustering is an effective way of organizing sensor networks. Among all clustering grid based clustering is important and efficient due to its scalability.

\subsection{Clustering Schemes}

A WSN consists of hundreds or thousands of densely populated sensor nodes that sense the environment i.e. sense the data and propagate through the environment. They are work collaboratively to process and route sensor data. These sensor nodes send data streams to base stations either periodically or based on events and base station send the data to the destination node. In a network densely populated with sensor nodes, the area detected by the sensors may overlap, and thus the data sensed by the sensors may be similar. Many wireless collisions occur in this type of network.

In clustering scheme network is grouped into different clusters Each cluster is composed of one cluster head $(\mathrm{CH})$ and cluster member nodes. The respective $\mathrm{CH}$ gets the sensed data from cluster member nodes, aggregates the sensed information and then sends it to the Base Station. [6]

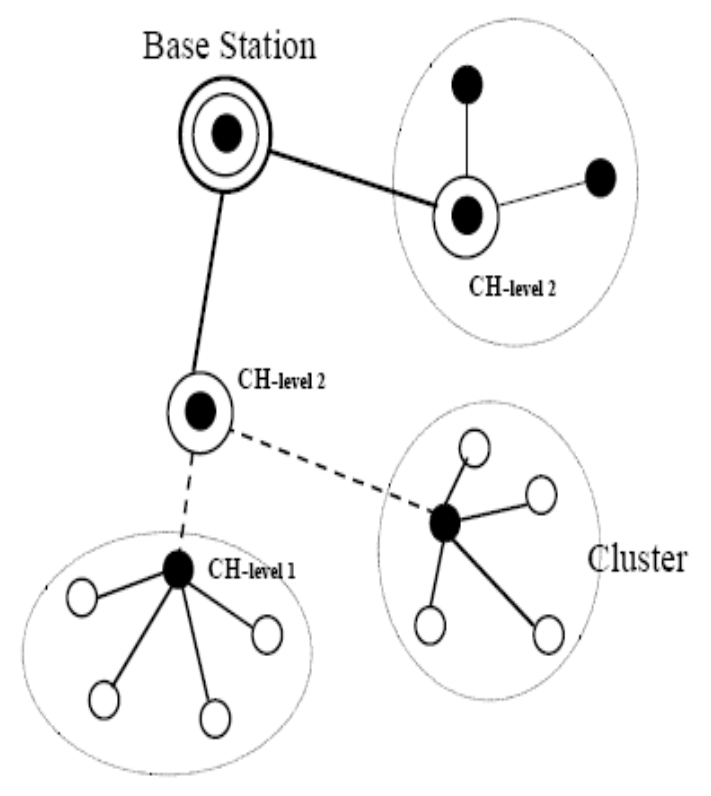

Fig 3. Cluster based model

In clustering schemes, sensor nodes are dividing into a number of small clusters. Each cluster has a coordinator or master head i.e. the cluster head $(\mathrm{CH})$, and a number of cluster nodes. Clustering results in a two-tier hierarchical structure, in which if cluster node want to send data to the sink node, then instead of sending directly, cluster nodes first transmit data to their own $\mathrm{CH}$ directly, while $\mathrm{CHs}$ collect the data and send them to the sink node through the $\mathrm{CHs}$ in other clusters. Meanwhile, redundant cluster nodes can be put into the sleep mode, since sensors within the sense and transmission range of others have no need to be active all the time. Therefore, clustering schemes are widely used in wireless sensor networks, not only due to their simple node coordination, but also because they use multi-hop routing between $\mathrm{CHs}$ to avoid long-range transmissions.

\subsection{Cluster Head Selection}

Each cluster has a coordinator or master head i.e. the cluster head $(\mathrm{CH})$. For $\mathrm{CH}$ selection any algorithm can be applied with our work. In Cluster formation process, Firstly a cluster head is selected then with the collaboration of BS clusters are formed and finally routing is carried out. The cluster head selection phase starts and all the deployed nodes send their energy levels to the Base Station. Then on the basis of energy level, geographical area and least id cluster head are selected. Network deployment is considered as manual so the base station is well informed about the geographical locations of the nodes. Base Station will select the cluster heads and multicast this information to them. $\mathrm{CH}$ selection flow diagram is presented in figure 4.[6 ] 


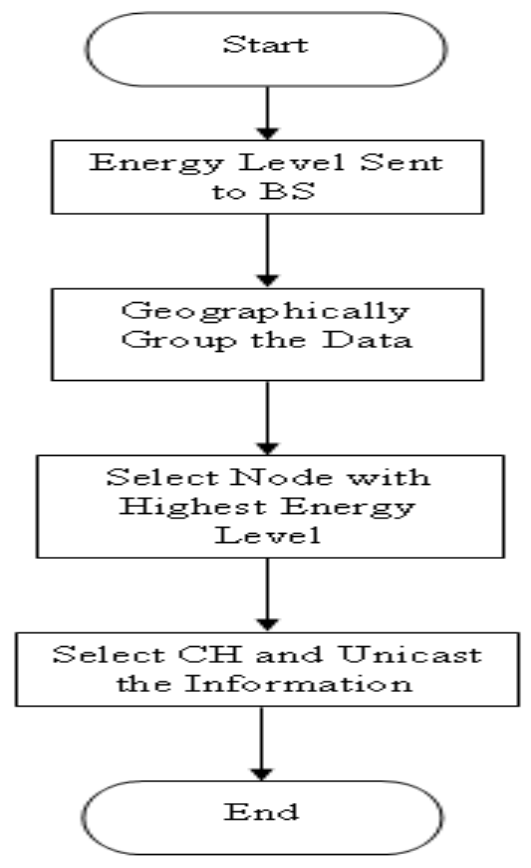

Fig 4: Flow diagram of $\mathrm{CH}$ selection

\section{DESIGN OF EXPERIMENT}

A WSN consists of hundreds or thousands of sensor nodes that are scattered in the environment. Node continuously sense the data and propagate through the environment. They are work collaboratively to process and route sensor data. These sensor nodes send data streams to base stations either periodically or based on events and base station send the data to the destination node. One of the problem in WSN is how to create an efficient organizational structure amongst these node. Since the fundamental advantage of WSNs is the ability to deploy them in an ad hoc manner, as it is not feasible to organize these nodes into groups predeployment. For this reason, there has been an large amount of research into ways of creating these organizational structures (or clusters).

There are three steps in the proposed scheme: grid based clustering, cluster-head election, and Intra Cluster Routing. The process of clustering first divides the network into grids. Based on the grid structure, it select the $\mathrm{CH}$ and after that perform cluster routing. Therefore constitute the layered structure shown in Fig. With a grid-based scheme, energy can be further conserved by a predefined route between the data source and the sink.

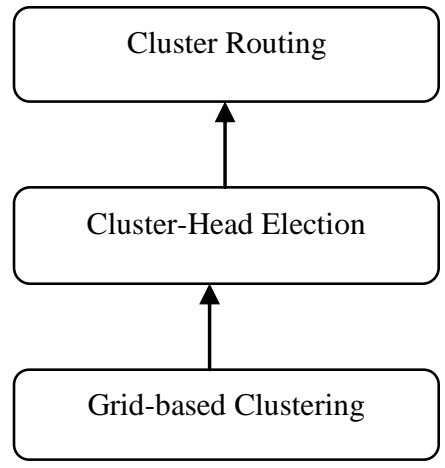

Fig 5: Layered Structure

\subsection{Grid based clustering}

Among all clustering Grid-based clustering and routing schemes, in which clusters are equally-sized square grids in a two-dimension plane, have a simple structure with less routing management overhead, and all nodes in one grid are equivalent from the routing perspective. With the assistance of GPS or localization techniques, the square grid also provides easier coordination among all sensor nodes in the network. Therefore, it allows for a theoretical analysis while still being useful enough to incorporate all the important elements of a network. Extensive research work has been done in grid-based clustering. In the early work of GAF, the grid size $\mathrm{s}$ is chosen such that any two nodes in horizontally or vertically adjacent grids are within the transmission range, $r$, of each other, which is referred to as Manhattan walk in Fig. 6 (a). By investigating the worst-case scenario, the grid size should be $\mathrm{s} \leq \mathrm{r} / \sqrt{ } 5$.

Recently, the work of also uses this clustering structure. For the one-dimension case, $\mathrm{s}$ should be less than $\mathrm{r} / 2$. More recent work of and used a smaller grid size, $\mathrm{s}^{\prime} \leq \mathrm{r} / \sqrt{8}$, allowing nodes in diagonal grids to be in the same transmission range as well, as shown in Fig. 6(b). With the same transmission range $r$, there are fewer grids in Fig. 6(a), but it may take more hops to reach the sink. Thus the tradeoff between these two gridding approaches is still an open question. [1][8]

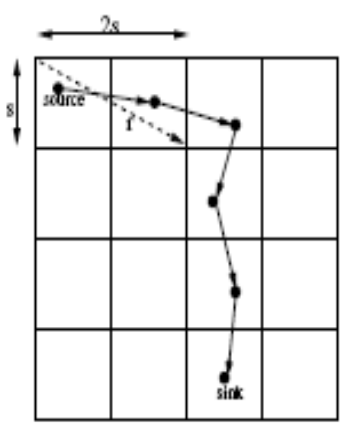

(a)

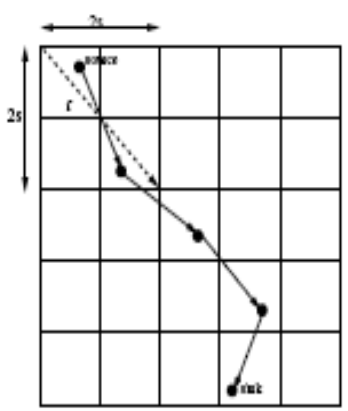

(b)
Fig 6: Manhattan walk $(\mathrm{s} \leq \mathrm{r} / \sqrt{ } \mathbf{5}) \&$ diagonal-first $(\mathrm{s} \leq$ $\mathbf{r} / \sqrt{ } \mathbf{8})$.

In our proposed clustering scheme network is grouped into different clusters, In this module we are implementing the grid based clustering algorithm depending on the interest of nodes. Here, firstly we are calculating the interest of node and depending on their interest, we are forming a cluster. Fig below shows the clustering of nodes.

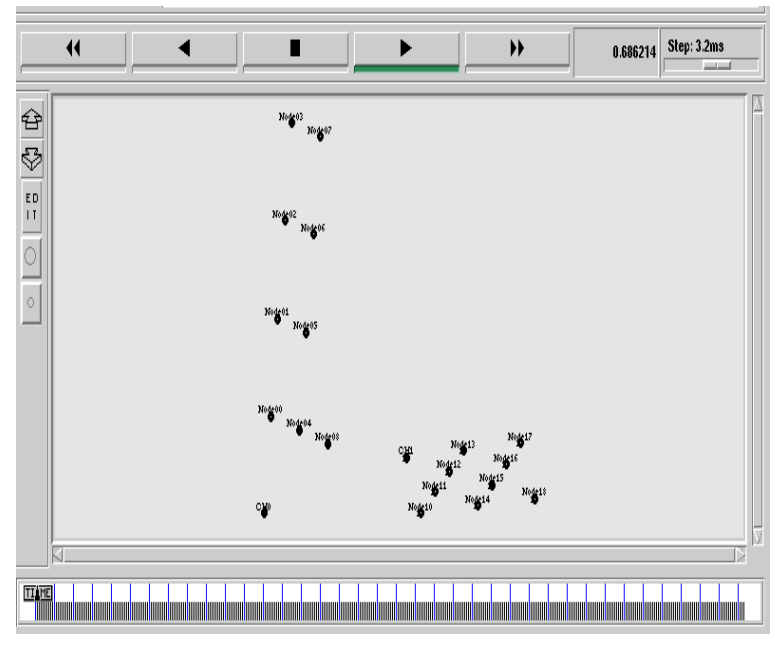

Fig 7:Showing Clustering of nodes 


\subsection{Cluster Head Selection}

Each cluster has a coordinator or master head i.e. the cluster head $(\mathrm{CH})$.For $\mathrm{CH}$ selection we can be applied any algorithm. In Cluster formation process, Firstly a cluster head is selected then with the collaboration of BS clusters are formed and finally routing is carried out.

In this module, Cluster Head is selected by comparing the weight of all the nodes present in a cluster. If the weight of a particular node from cluster is greatest as compared with other then that that particular node is considered as a Cluster Head. Fig. below shows the Cluster Head in a cluster.

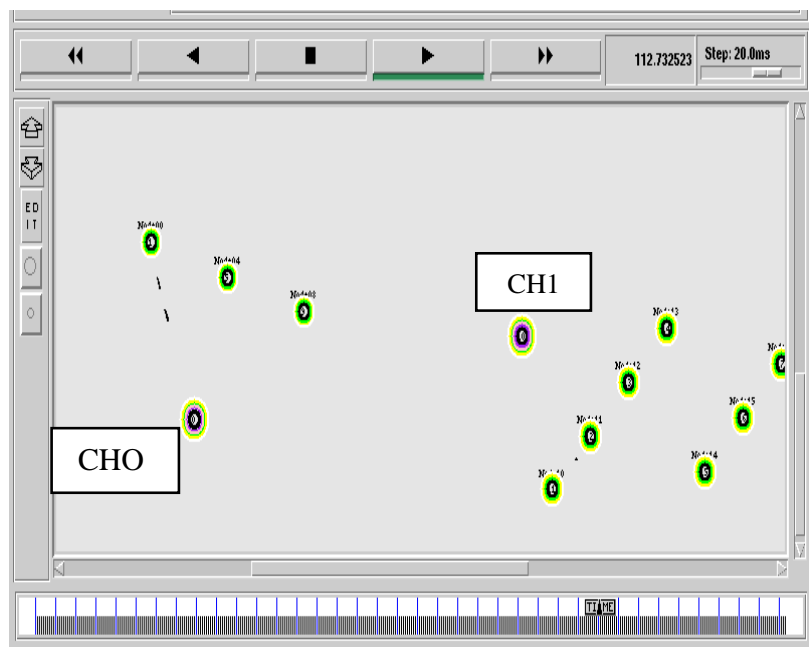

Fig 8: Showing Cluster Head

\subsection{Cluster Routing}

There are two types of routing- a) Intra cluster Routing b) Inter Cluster Routing. In intra cluster routing, data transmission takes place between the nodes in a same cluster. In Inter Cluster routing, routing takes place between the nodes of different cluster. Fig below shows the Intra cluster Routing.

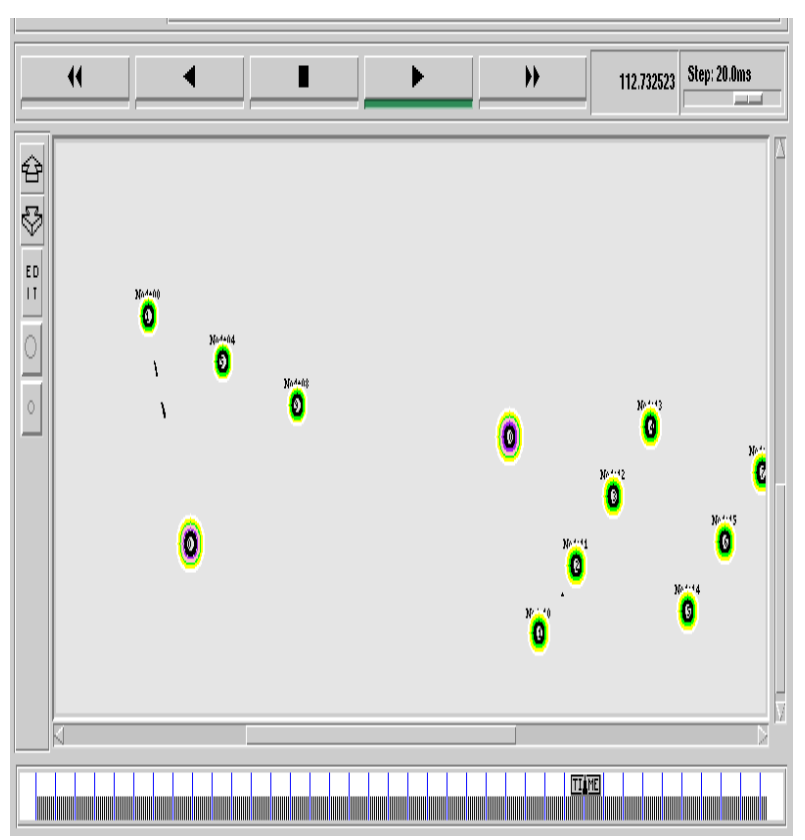

Fig 9: Showing Intra Cluster Routing
In this proposed scheme, we are using Drop-tail type of queue, which is present at each node. If the packets coming towards perticular nodes exceeds the limit of queue, then queue will drop the packets. Fig. 10 shows the dropping of packets by node.

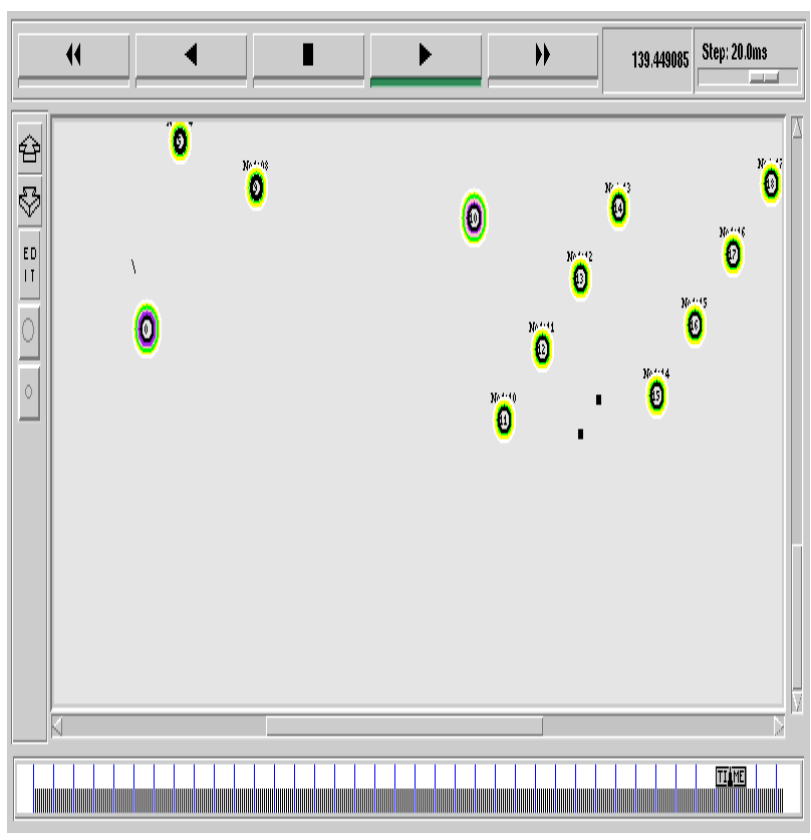

Fig 10: Showing packet drops by the node.

\section{ADVANTAGES OF GRID BASED CLUSTERING}

- Simple and feasible

- "Once the grid structure is established nodes can communicate locally with their grid head and reach the data processing center, or the sink node, through neighbor grids."

- They can transmit data directly, no explicitly path creation.

- Useful for Real-Time Application

\section{REFERENCES}

[1] Y. Zhuang, J. Pan and G. Wu, "Energy-optimal grid based clustering in wireless microsensor networks," IEEE ICDCS Workshop on Wireless Adhoc and Sensor Networking (WWASN), 2009.

[2] R. Vidhyapriya and P. T. Vanathi, "Energy efficient gridbased routing in wireless sensor networks," International Journal of Intelligent Computing and Cybernetics, vol. 1, no. 2, pp. 301-318, Jan. 2008.

[3] K. Akkaya, M. Younis, "A survey on routing protocols for wireless sensor networks," Ad Hoc Networks, vol. 3, no. 3, pp. 325-349, May 2005.

[4] Chin-Ling Chen and I-Hsien Lin, "Location-Aware Dynamic Session-Key Management for Grid-Based Wireless Sensor Networks particles, thin films and exchange anisotropy," Sensors 2010, 10, 7347-7370; doi:10.3390/s 100807347. 
[5] Ameer Ahmed Abbasi and Mohamed Younis, "A survey on clustering algorithms for wireless sensor networks," Computer Communications 30 (2007) 2826-2841.

[6] Adeel Akhtar, Abid Ali Minhas, and Sohail Jabbar , "Energy Aware Intra Cluster Routing for Wireless Sensor Networks," International Journal of ybrid Information Technology Vol.3, No.1, January, 2010.
[7] Sylvain Ranvier , "Path loss models," Radio Laboratory TKK 23 November 2004.

[8] Yanyan Zhuang ,Jianping Pan and Lin Cai, "Minimizing Energy Consumption with Probabilistic Distance Models in Wireless Sensor Networks," IEEE INFOCOM 2010. 